\title{
Tweet me
}

\author{
Meeting organizers and speakers are increasingly open to blogging and microblogging - an \\ encouraging trend that should be expanded with clearly defined restrictions.
}

Blogs and microblogs, such as Twitter and FriendFeed, have added a new dimension to interpersonal communication. Talks at scientific meetings are not exempt from this trend. Over the past two years it has become increasingly common to find summaries of talks posted on scientists' blogs or salient points discussed on microblogs. With some exceptions, meeting organizers support this development and leave it up to presenters to impose restrictions. We support this model but encourage speakers not to set unnecessary limits.

Social media provide a valuable service to the scientific community and, to a certain extent, also to presenters. Scientists are always interested in more meetings than they can physically attend, and following blogs is a good way to keep up to date. Even meeting attendees benefit from blogs: sessions often run concurrently, question-and-answer time is limited, and only so many scientists can gather in a bar.

For speakers, blogging will increase the awareness of the work presented and may reveal open questions or flawed reasoning, and such scrutiny is one of the reasons to present the work in the first place.

What are presenters' main concerns about having their work discussed in social media? Worries about getting scooped after presenting unpublished data are probably high on the list. But how much will a blog increase the likelihood of this happening? There is an inherent risk of getting scooped after publically presenting new data. Speakers can try to gear their message to a particular audience - something that is impossible if one has to consider the more global readership of blogs. But how much control does a presenter really have over who ultimately hears a message? Unexpected guests may be in the audience, and word of mouth will take content to people beyond a lecture hall.

Some may fear that coverage in a blog could be construed as prepublication. This seems unlikely because journals do not count talks as prepublication, and unsolicited coverage in blogs also falls under this category.

Another concern is that the work needs to be read in the context of a fully peer-reviewed article and that premature release would cause confusion among a wider audience, for example, in large-scale medical studies reporting the efficacy of certain treatments. This concern has merit and warrants a blogging restriction.
It bears remembering what a blog is, or rather, what it is not. Blogs are not live streams of talks or a replacement for peer-reviewed articles but an extension of the scientific discourse. They contain summary notes that announce the main findings and direction of the work and provide room for comments by readers. Microblogs are limited to 140 characters, within which one can do little more than repeat salient points, raise questions or present relevant links.

Whereas blogs do have the potential to give inaccurate or incomplete account of scientists' work, it is in the bloggers' best interest to present facts accurately and clearly indicate when personal opinions are presented. Scientific bloggers want to retain their reputation by providing a service rather than food for gossip. Helpful guidelines for blogging etiquette can be found in PLoS Computational Biology (doi:10.1371/journal. pcbi.1000563).

An increasing number of meeting organizers welcome bloggers and encourage the coverage of talks. Others, such as those at Cold Spring Harbor Laboratories and Janelia Farm, have installed additional filters requiring bloggers to gain permission from a presenter before writing about a talk.

Putting the onus on the blogger, however, unnecessarily impedes the flow of information. Instead, presenters should clearly state which part of the talk should be exempt or whether the whole talk is embargoed.

Organizers of the recent Advances in Genome Biology and Technology meeting experimented with this model. Restrictions were generally clear and reasonable: for example, bloggers were asked to withhold patient data. What caused some discontent was the fact that some presentations covering published data or topics that need community input were barred for bloggers; this seemed arbitrary and stifling to a fruitful discussion.

It is important to have an honest discussion about blogging policies to ensure that the needs of the scientific community for information are met while addressing a presenter's concern about premature exposure of sensitive work.

We are keen to hear your views on the subject on our blog, Methagora, and we invite you to follow us on Twitter@naturemethods. 